\title{
Research and application upon Open ed- ucation graduation thesis database system based on web 2.0
}

\author{
Zhenxiang Liu ${ }^{1}$ Xiaolin Xin ${ }^{1}$ Yan Jia ${ }^{1}$ Ping Yu ${ }^{1}$ Xinran Wei $^{2}$ \\ ${ }^{1}$ Guizhou Radio and TV University \\ ${ }^{2}$ Guizhou University
}

\begin{abstract}
Aiming at repairing the shortcomings of the existing Open education graduation thesis database system, we develop an Open education graduation thesis database system based on web 2.0 according to the notions and methods of Web 2.0.Ajax, LINQ, Social labels and other technique are introduced into our system. Our system can be promoted to National Open University system because it has the characteristics of modern literature resources system: system resources platform is open and user-centric.
\end{abstract}

Keywords: Open Education; graduation thesis; Web2.0; Ajax; Social labels

\section{Introduction}

China open education is centre on serving officers with the usage of Modern means of information technology Distance advanced Education. Open education system is sponsored by the Open University of China, and 44 provinces (autonomous regions and municipalities directly under the Central Government) Open University. Currently there are more than 70 of the specialist professional and more than 300 million students in school, and obviously it is an important part of Chinese advanced education.

Graduation theses are important results of the students' study, and they can di- rectly reflect the comprehensive strength and characteristics of the Open University in teaching, research and personnel training. Meanwhile, Open education graduation theses are also significant literature resources due to their reference and academic value in the study of related disciplines.

The number of graduation thesis is increasing, because there are more and more students are accessing open education in national Open University. However, the situation inspected from the national well-known databases is unsatisfactory. Obviously national Open University's teaching and scientific research are not able to be demonstrated from the coverage of these databases. Consequently it can not play its due role. The main reason is that current major open education thesis databases are simple digital processing. This leads to the leakage of the basic functions of the network literature resources system. Even those databases equipped with the basic functions of network database system are based upon Web 1.0, which result to the closure of the system and the underutilized resources.

Aiming at the deficiency of existing open education thesis database and according to the notions and method of Web2.0, the research of open education graduation thesis database system based on Web 2.0, to realize the resource platform open, take the customer as the cen- 
ter, pay more attention to the user experience, enable users to participate in the system construction, and create new value for resources, is the need to the conservation and full use of open education thesis resources, and is also the need to further improve the quality of teaching.

\section{System Design}

\subsection{Functional Design}

The function division of the system is shown in Figure 1: user management, resource management, comments management, collections management, after-user management, background management.

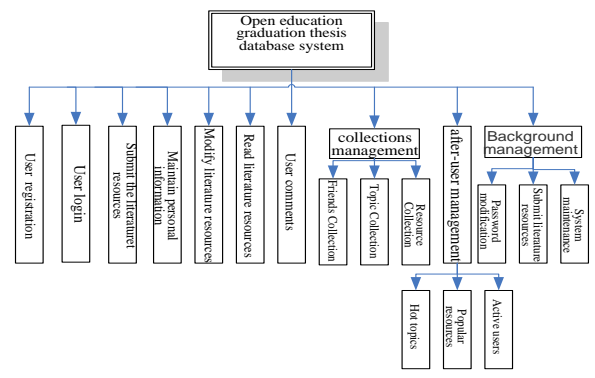

Fig. 1: Graduation thesis database system function design.

\subsection{Database design}

The data types of the system include: resource file (degree thesis), social tags, user information, the log, comments, personal collection etc, divided into three entities: User (readers, administrator), Discourse, Comments (upload, download, comment).

Data items that User entity involved include: Account, ID, Name, Password, Sex, Education, Unit, Phone, NumQQ, Email, and Remark, Figure 2 is User information DDMS entity figure.

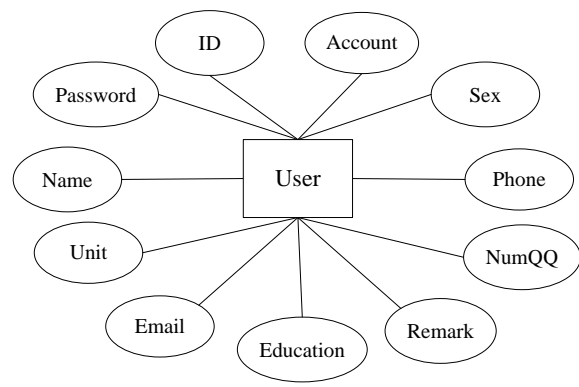

Fig. 2: User information DDMS entity figure.

Data items that the Discourse entity involved include: ID, Title, Author, Summary, Key Word, File, Type, Upload User, Date, and Audit, figure 3 is the Discourse information DDMS entity figure.

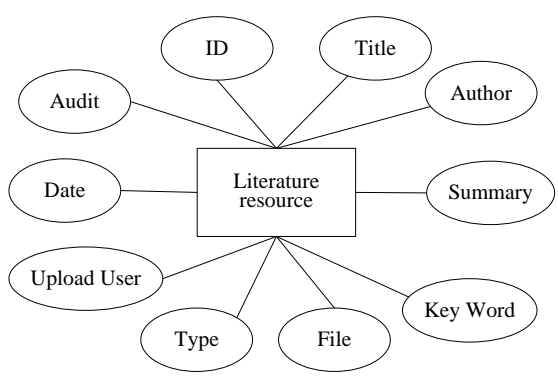

Fig. 3: Discourse information DDMS entity figure.

Data items that the Comments entity involved include: Comment_ID, Resource_ID, User_ID, Content, and Time, figure 4 is the Comments information DDMS entity figure.

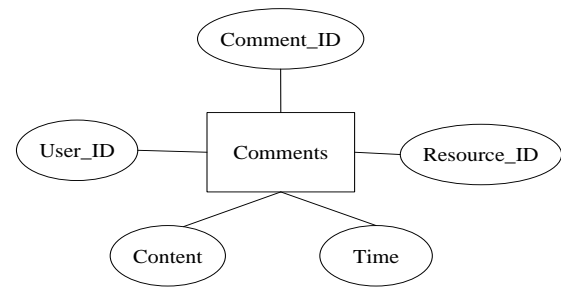

Fig. 4: Comments information DDMS entity figure.

2.3. Entity class relations design 
(1) The Discourse and File mapping relationship

Every literature resources must contain its own description and a source file, the source file needs a description of the relevant information on the file itself. As shown in Figure 5, the definition of two classes to describe that the File class is included in the Discourse class, which is one of the components of the Discourse.

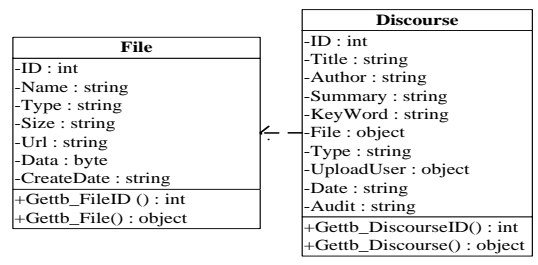

Fig. 5: File and Discourse class figure.

(2) Tags relationship design

We should paste thesis suitable tags when to submit theses and theoretically speaking the number of tags is uncertain. So there can be more than one tag. The tags should be part of the thesis information, but different thesis will have the same tag, both of which reflect a manyto-many relationship in the database. Then we use three tables to store them for more convenient data maintenance management. Specific storage conditions as shown in Figure 6.

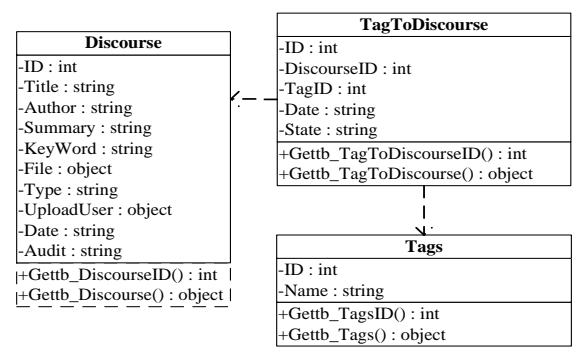

Fig. 6: Tags class figure

(3) MyCollect relationship design

Three of the content system of friends (user), the tag (topic), resources (theses), are stored in table three, because the data structure will be the same, and indicates the type of field collection of the last, the three together, in order to facilitate the management. Specific design as shown in Figure 7.

\begin{tabular}{|l|l|}
\hline \multicolumn{1}{|c|}{ MyCollect } \\
\hline -ID : int \\
-User : object \\
-CollectID : int \\
-CollectType : string \\
-Date : string
\end{tabular}

Fig. 7: MyCollect class figure

\section{System Implementation}

The system is based on Windows operating system, database uses SQL Server 2005 Developer Edition (32 bit), and development tools are Microsoft Visual Studio 2008 and C \#.

The system uses Ajax technology to reduce the bandwidth occupancy through the asynchronous mode, to give the user the fastest feel and experience and make the page more human. The system adopts three layer architecture, which makes the system structure clear, makes development and maintenance workload small, and makes the function point relatively concentrated. At the same time, the introduction of LINQ technology makes data merging more quickly and easily. The following will analyze several typical function implementations.

\subsection{Thesis Management}

We need to fill out the feature information and add custom tags when to submit thesis. And when to upload to the 
server, we need to create a new file for the thesis, read the file's name, size, type, and other information. In order to ensure the uniqueness of the name, the current time is converted to a string, as the file name on the server. The process is shown in Figure 8.

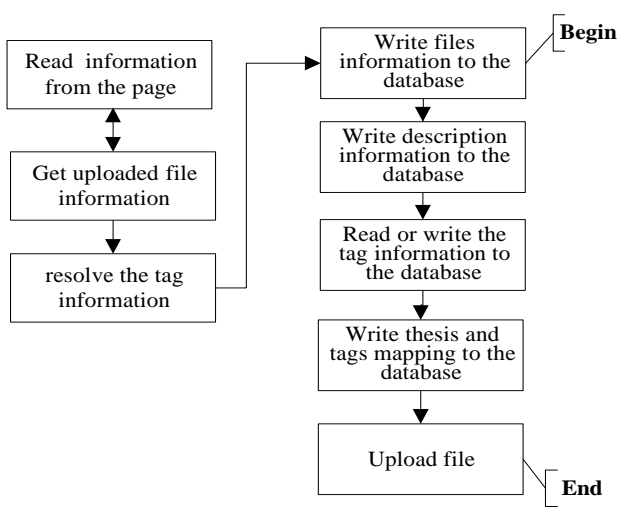

Fig. 8: Submission process figure

As shown in Figure 8, the system uses the transaction mechanism in submission process, in which any steps appear problem, the operations will return to the previous state.

\subsection{Tags Application}

Generally, the creation of the tags is added by the user when to submit papers, and it divided into single tag and multiple tags. The addition of a single tag is that the tag text is submitted to the table Tags, at the same time, the tag ID is recorded in the Tag field of the table content. Then to add multiple tags, we can split the tag text into several separate tags by tags separator, then press the single label processing.

When to search a thesis, tag ID as the condition is queried. If it meets the conditions of, system will generate a data collection, and then the records of the data collection will be displayed by a loop statement. In addition to finding thesis by tags, users can also find other users on the same tag content, methods are as follows:

The first step, the tag as the condition to check out all the papers included the label in the content table;

The second step, there is a Author field holding the author name in the query records. In these records, it is likely that the same authors published many articles, so the record set is classified, and we can draw all users who use the tag.

The third step, after we get all users who use the same tag, then we can also get the users' links, in order to access the pages of these users and realize the communication between users.

\subsection{Collection Management}

Friends Collection: Users can select friends they would like to collect to add to their friends list. The system provides all non-collection of user list information. Then users can choose to add according to their needs. When one adds a friend, the added party will receive a notification message which shows that he or she is added as a friend by others. Similarly, the added party could add the other side to the friends list. After one adds friends, he can see all dynamic information about his friends through his friends' hyperlinks.

Topic Collection: Users can select the tag involving the topic their own interest by tags list. The system provides all noncollection of tags list information. Then users can choose to add according to their needs. After one adds tags, he can see all resource information about the topic through hyperlinks.

Resource Collection: Users can collect the meaningful and valuable literature resources to their resources list. The system provides all non-collection of literature resources list information. Then users can choose to add according to their needs. When one collect literature resources, the original author of the resources will receive a notification mes- 
sage which shows that his resource is collected by others and displays the collection party information.

\subsection{After-user Management}

Popular resources: The user interface can display existing resources according to the user access frequency from high to low. Once the user accesses data information, the data information will be marked the number of visits. The more visits, the larger the degree of concern.

Hot topics: The topic is the literature resources tab. The same resources will have several tags, so the same resources can belong to a different topic, and a topic also contains a lot of resources. The topic popularity is reflected in the number of resources involved in this topic. The more resources related to this topic, the more popular.

Active users: The system can calculate the rank of users, in accordance with their login frequency. Once a user logins in the system, his login information can been recorded in the log information table. Login times of individual user can be counted through login table and then sort the results. Finally user login frequency can be obtained.

\section{Application Analysis}

Based on the need of the open education thesis database system construction, management and usage, we develop the open education graduation thesis database system based on web 2.0. And it is under trial at the Open University of Guizhou for two years. The teachers and students fully affirm its significance and effect. Compared with the previous thesis database, this system has the typical characteristics of modern literature resources system, and embodies in the following.

- The system interface is simpler and more humane what gives users convenient and friendly feeling and experience.

- Authorized users can freely and easily upload, download and maintenance graduation thesis;

- The system adopts the social tags to classify the graduation thesis to provide a more complete and effective information classification mode. In addition, the search based on the social tags and the user collections facilitate users query and search, to improve personalized, practical and sharing;

- Users can collect their favorite contents and authors, and collect the key or interest points of the cyber source content, to understand the readers' real perception;

- Users can discuss with others about their favorite contents.

- In order to push valuable information for users, the system can automatically record the traces of users browsing system and information query clues.

In addition, through collecting and collating thesis and detailed reviews of instructors, the system plays a catalytic role in counseling for students in learning, accumulating teaching achievement and improving teaching quality for university.

\section{Conclusion}

Based on the present situation of literature construction, management and usage of Open University graduation thesis, we focus on the shortage of Open University graduation thesis database system when study, develop and use it. In order to realize quickly and effectively service for teaching, research, and even economic construction and social development on the Interne we develop a new generation of Open University graduation thesis database system to manage such a large number of important literature resources. 
In the two years of the trial, the results show that Open University graduation thesis database system has achieved the goals, equipped with typical characteristics of modern literature resources system and received well feedback from teachers and students. Furthermore, this Open University graduation thesis database system can be promoted to National Open University system.

\section{References}

[1] Fan Mo, "Common Morphology and Propagation Characteristics of Web2.0", Journal of Kaifeng University, pp. 77-78, 2006(2).
[2] Runhua Wang, Xiaoyan Yun, and Minghua Du, "The Wiki and its Application to Teaching Education", Journey of Educational Technology, pp. 4-5, 2005(5).

[3] Shuren Zhang, "From Social Software, Web2.0 to Complex Adaptive Information Systems Research", School of Information Renmin University of China, 2006.

[4] Sen Lin, "Knowledge of Personalized Recommendation and Systems based on the Tag technology", Huazhong University of Science and Technology, 2006.

[5] Phil Ballard, "Sams Teach Yourself Ajax in 10 Minutes", Addison-Wesley, pp. 1-200, 2006. 